\section{Avaliação do uso de albumina humana em hospital do Rio de Janeiro, Brasil}

\author{
Evaluation of human albumin use \\ in a Brazilian hospital
}

Guacira Corrêa de Matos 1

Suely Rozenfeld 1

\footnotetext{
1 Escola Nacional de Saúde Pública, Fundação Oswaldo Cruz, Rio de Janeiro, Brasil.

Correspondência G. C. Matos

Departamento de

Epidemiologia e Métodos Quantitativos em Saúde, Escola Nacional de Saúde Pública, Fundação Oswaldo Cruz. Rua Leopoldo Bulhões 1480, Rio de Janeiro, $R J$ 21041-210, Brasil. gmatos@matrix.com.br
}

\begin{abstract}
Human albumin is frequently used without regard for the relationship between its pharmacological properties and the respective disease. We investigated the prescription of human albumin in a Brazilian public hospital with emphasis on adherence to international guidelines. Data were gathered from pharmacy and medical records. From March to August 2001, 99 patients ( $\geq 15$ years) received 1,475 units of human albumin, ordered in 498 prescriptions. The reason for prescribing human albumin was independently collected from medical records by two health professionals, and agreement was assessed by kappa statistics (95\%CI: 0.72-0.85). According to different guidelines, prescriptions were classified as appropriate (33.1\%), inappropriate (61.8\%), controversial (4.6\%), or undetermined (0.4\%). Two probable cases of adverse reactions were identified. The proportion of inappropriate prescriptions is worrisome due to the product's high cost and its potential to cause adverse reactions. An appropriate strategy would be to encourage the application of guidelines issued by the national regulatory agency to promote rational use of human albumin and maximize patient safety.
\end{abstract}

Serum Albumin; Drug Utilization; Services Evaluation

\section{Introdução}

A albumina humana é um produto de ampla utilização e de custo elevado. O consumo mundial, de cerca de seiscentas toneladas por ano, corresponde a um gasto de mais de US\$ 1,2 bilhão 1 . Está disponível no mercado brasileiro em solução injetável nas concentrações de 20 a $25 \%$, e existem mais de cinqüenta registros do produto no Ministério da Saúde (MS), entre importadores e produtores 2 .

No Brasil, a administração de albumina humana integra o elenco de procedimentos especiais do MS 3 . O consumo interno atinge a mais de $10.000 \mathrm{~kg}$, sendo que a produção nacional atende a menos de 10,0\% do total. Atualmente, o MS desenvolve um projeto de implantação de unidades industriais públicas, que visa a atingir auto-suficiência em hemoderivados, utilizando o plasma excedente dos Serviços de Hemoterapia do país 4.

A albumina humana é um medicamento hemoderivado injetável, extraído do plasma humano, desenvolvido na década de 1940. A partir da coleta de sangue humano são obtidos os produtos sangüíneos lábeis (hemocomponentes), por fracionamento do sangue total, e os produtos sangüíneos estáveis (hemoderivados), que passaram por fracionamento e processos de purificação e estabilização industriais. Dessa forma são produzidos os hemoderivados não anti-hemofílicos, como a albumina humana, e os anti-hemofílicos 5 . 
A albumina é considerada um produto seguro quanto à transmissão de doenças infecciosas, desde que garantidas as boas práticas de fabricação, armazenamento e administração. Na fase de fabricação, o produto é submetido a um processo de esterilização por pasteurização a $60^{\circ} \mathrm{C}$ por dez horas. Entretanto, subsiste sempre um risco potencial de transmissão de vírus e de partículas subvirais e, atualmente, há estudos que visam ao aperfeiçoamento do processo de inativação por nanofiltração 5,6.

As indicações terapêuticas da albumina humana estão, em sua maioria, relacionadas à reposição de perdas volêmicas agudas e à manutenção da pressão coloidosmótica do plasma. Em muitos casos é recomendada a substituição terapêutica da albumina humana por expansores plasmáticos sintéticos, que reduzem a chance de efeitos adversos e os custos de tratamento. Os principais expansores sintéticos são as soluções cristalóides (cloreto de sódio, Ringer) e os colóides não protéicos (dextrana, poligelina, amido hidroxetil) 7,8 .

As atualizações mais recentes de duas revisões sistemáticas de ensaios clínicos, realizadas pelo Cochrane Injuries Group Albumin Reviewers, não encontraram evidências de que os colóides sejam mais efetivos ou seguros do que os cristalóides na ressuscitação e na expansão de volume em pacientes críticos 9,10. Outra revisão Cochrane 11, atualizada em 2004, não encontrou evidências de benefícios do uso da albumina humana na redução da mortalidade de pacientes críticos com hipovolemia, queimaduras e hipoalbuminemia, quando comparado com o uso de outros expansores plasmáticos. O maior peso do resultado dessa revisão advém de um grande ensaio clínico randomizado, realizado na Austrália e Nova Zelândia, com 6.997 pacientes, que comparou a efetividade da albumina $4,0 \%$ e da solução salina $0,9 \%$ como fluidos de ressuscitação e não encontrou evidências de vantagens de um tratamento sobre o outro 12 .

Desde meados da década de 1970, instituições e pesquisadores de diversos países desenvolvem protocolos práticos para a racionalização de indicação e prescrição da albumina humana 7,13. Em 1975, o National Institute of Health (NIH), Bethesda, Estados Unidos, realizou uma conferência de consenso para desenvolver um protocolo de uso da albumina humana 14,15. O estudo, publicado em 1977, foi, durante duas décadas, a base de protocolos de vários hospitais, de estudos clínicos, artigos de revisão, livros-texto e da prática terapêutica corrente 7 .
Entretanto, mesmo vários anos após a adoção dos protocolos, o nível de adesão não atinge taxas elevadas, mantendo-se alta a proporção de prescrições em desacordo com as diretrizes 8,16. Esse problema, comum a todos os tipos de protocolos práticos, está ligado a fatores como a resistência à interferência na prática clínica, o emprego de estratégias inadequadas ou insuficientes de adesão, a rigidez da condução terapêutica proposta e os conflitos de interesses 17,18

Estudos observacionais realizados em diversos países encontraram cerca de 10,0 a 30,0\% de prescrições em conformidade com as diretrizes dos protocolos adotados. Em muitos casos, não havia contra-indicações para o uso dos substitutos terapêuticos da albumina humana ou ainda, os pacientes não necessitavam qualquer tipo de expansor plasmático. Em todos os estudos foram evidenciadas as repercussões econômicas do uso irracional da albumina humana 8,13,16,19,20.

No Brasil, os estudos de utilização da albumina humana são escassos, embora existam algumas iniciativas isoladas de adequação e controle do uso da albumina humana 21,22. A Agência Nacional de Vigilância Sanitária (ANVISA), publicou em 11 de maio de 2004, a Resolução de Diretoria Colegiada (RDC) n. 115, que estabelece diretrizes para o uso terapêutico da albumina humana 23 .

O estudo das características do consumo e da prescrição da albumina humana pode fornecer importantes subsídios para o delineamento de ações que visem à racionalização do seu uso e a efetiva implantação do protocolo nacional.

O presente estudo pretende descrever e analisar, criticamente, as características do uso de albumina humana em hospital selecionado da rede pública de saúde, localizado no Estado do Rio de Janeiro, Brasil.

\section{Método}

\section{Método, população e fonte de dados}

Este é um estudo seccional realizado em hospital geral da rede pública federal de saúde no Rio de Janeiro, com mais de 440 leitos, distribuídos em vinte clínicas. Os critérios de seleção do hospital foram: o perfil assistencial, o quantitativo de consumo de albumina humana e a viabilidade de acesso aos dados. O elevado consumo anual de albumina humana vem sen- 
do alvo de preocupação da direção do hospital. Em 2001, segundo os registros do Serviço de Farmácia, foram consumidas 7.980 unidades de $50 \mathrm{ml}$ de albumina humana $20,0 \%$, correspondendo a um gasto de $\mathrm{R} \$ 344 \mathrm{mil}$. O Serviço de Farmácia atende os pedidos de albumina humana por meio de prescrições diárias por paciente e por clínica, o que permite a localização dos prontuários médicos no arquivo do Serviço de Documentação Científica, usandose o sistema informatizado de busca.

A população do estudo é constituída por adultos, com 15 anos ou mais, internados nas enfermarias, no período de março a agosto de 2001. As crianças abaixo de 15 anos foram excluídas, com o objetivo de tornar a população do estudo mais homogênea. Também foram excluídos os adultos internados no CTI porque são pacientes críticos, com quadros clínicos complexos e polimedicados, o que torna a análise da adequação de uso controvertida e imprecisa. Os pacientes da emergência também foram excluídos, porque, em sua maioria, não possuem prontuário médico, impossibilitando o acesso aos dados.

\section{Coleta de dados}

Os dados foram obtidos dos registros de controle de entrada de albumina humana no Serviço de Farmácia, dos registros diários de prescrição de albumina humana do Serviço de Farmácia e dos prontuários médicos dos pacientes que receberam albumina humana, durante o período estudado, e que atenderam aos critérios de inclusão previamente estabelecidos.

As informações coletadas a partir dos registros de controle de entradas no Serviço de Farmácia foram consolidadas no Mapa de Registro de Entradas de Albumina Humana 20\% $50 \mathrm{ml}$ e tornaram possível conhecer o quantitativo, a procedência e o custo unitário.

Nos registros diários de prescrição do Serviço de Farmácia de 2001, obtiveram-se as informações sobre os pacientes e as clínicas e os quantitativos individuais diários prescritos e dispensados. Como não havia número de prontuário anotado, esse dado foi obtido com o Serviço de Documentação Científica. Os registros do Serviço de Hemoterapia foram consultados, complementarmente, para facilitar a identificação dos números de prontuário, uma vez que é freqüente a prescrição de hemocomponentes e de hemoderivados para um mesmo paciente. Os dados extraídos deram origem à tabela dos quantitativos de dispensação de albumina humana, referente ao ano de 2001, discriminados por clínica, paciente, unidades, prescrições e data. Com base nesses dados foram gerados, também, os cadastros nominais de pacientes para os quais foi dispensada albumina humana, durante o período do estudo.

Dos prontuários médicos dos pacientes selecionados, de acordo com os critérios de inclusão e exclusão estabelecidos, foram extraídas as informações relativas ao paciente e às variáveis identificadas como necessárias para o estabelecimento das características do uso da albumina humana. Para tanto, foram desenvolvidos o Formulário de Coleta de Dados de Prontuário e o respectivo Manual de Instruções de Preenchimento, cujo processo de elaboração passamos a descrever abaixo.

As variáveis e respectivas categorias foram selecionadas com base nas utilizadas nos estudos realizados no Hospital Tenon, em Paris, França 20, e em hospitais universitários americanos 19 e espanhóis 8.

As variáveis foram agrupadas em dados gerais, dados clínicos, hábitos, exames complementares, terapêutica, reações adversas, indicação terapêutica e sinais complementares.

Uma versão preliminar do formulário foi testada com 13 prontuários, buscando identificar problemas quanto ao formato e funcionalidade e quanto à adequação das variáveis aos objetivos do estudo.

Em seguida, foram incorporados ao trabalho quatro alunos do curso de especialização em vigilância sanitária da Escola Nacional de Saúde Pública, Fundação Oswaldo Cruz (ENSP/ FIOCRUZ), que aplicaram a segunda versão do formulário e a primeira versão do manual de preenchimento. Esse piloto foi realizado em 31 prontuários do período de estudo. As informações de cada prontuário foram coletadas duas vezes, em formulários separados, por pesquisadores diferentes, para aperfeiçoar o manual de instruções e verificar a confiabilidade no tocante à indicação terapêutica. A versão final do formulário foi submetida a um especialista em hematologia e hemoterapia para avaliação.

A coleta definitiva foi realizada por dois pesquisadores contratados, ambos farmacêuticos, treinados pela investigadora principal. Antes do início da coleta foi feita a reordenação de cada prontuário por data e setor (evolução clínica, registros de cirurgia, registros de enfermagem, exames complementares). Os prontuários foram solicitados, por ordem de data, de acordo com a listagem, ao Serviço de Documentação Científica. Foram registrados os motivos da não localização de alguns prontuários. Todos os formulários preenchidos e os respectivos prontuários foram revisados pela responsável pelo estudo. 


\section{Análise}

As informações foram digitadas no programa Epi Data versão 3.0, duplicadas e validadas. As análises foram feitas no programa SPSS versão 10.0. As variáveis quantitativas foram expressas em média, desvio padrão e mediana, e as variáveis com distribuição não paramétrica em média, desvio padrão, mediana e intervalo interquartil (IQ). O teste $\chi^{2}$ foi usado para avaliar a associação da adequação da prescrição com o tipo de clínica (cirúrgicas e não cirúrgicas).

As indicações terapêuticas foram avaliadas como apropriadas, inapropriadas e controversas, de acordo com a Síntese de Protocolos (Tabela 1), que foi elaborada com base em quatro protocolos padrão de países diferentes, desenvolvidos por consenso de especialistas e baseados em evidências 7,8,20,23. Os casos em que não foi possível determinar a indicação terapêutica, com base no prontuário, foram classificados como indeterminados.

\section{Aspectos éticos}

O principal procedimento recomendado para garantir o respeito aos princípios éticos é a anuência dos sujeitos da pesquisa, por intermédio do Termo de Consentimento Livre e Esclarecido. Devido à inviabilidade de obtenção dos termos de consentimento individuais, pela impossibilidade de localização dos pacientes, a autorização para acesso aos documentos foi obtida da direção do hospital.

O projeto do estudo foi aprovado pelo parecer n. 110/02 do Comitê de Ética em Pesquisa da ENSP/FIOCRUZ.

Os pesquisadores assinaram um Termo de Compromisso de Utilização de Dados, onde se comprometem a preservar a privacidade dos dados, em somente utilizá-los para a execução do projeto e a divulgar os resultados de forma anônima.

Do formulário de coleta de dados constam informações de identificação dos pacientes: nome, número de prontuário, data de internação e data de alta ou óbito. Não há identificação dos prescritores. A investigadora principal conferiu os dados coletados e destacou a primeira parte dos formulários, onde constam os dados identificados e o número de ordem do formulário, mantendo-os sob sua guarda pessoal. Esses dados foram digitados pela investigadora principal em computador não ligado à rede de informática e salvos em discos flexíveis, que também ficaram sob sua guarda exclusiva. Ao seguir para a digitação, restaram apenas os dados não identificados e o número de ordem, repetido na segunda parte do formulário.

\section{Resultados}

Entre março e agosto de 2001, a farmácia dispensou 3.521 unidades de albumina humana para 308 pacientes das diversas clínicas, incluindo o CTI, a emergência, a UTI infantil e a pediatria.

Foram identificados, baseando-se nos registros da farmácia hospitalar, 115 pacientes que atendiam aos critérios de inclusão e para os quais foi prescrita e dispensada albumina humana, durante o período do estudo. Houve 16 perdas, cujos motivos estão detalhados na Tabela 2, restando 99 pacientes.

A confiabilidade entre pesquisadores da coleta da indicação terapêutica, realizada na fase piloto do estudo pela estatística kappa, foi de 0,79 (IC95\%: 0,72-0,85).

No total da amostra de pacientes, 56 eram homens e 43 mulheres. Quanto ao desfecho da internação, 67 obtiveram alta e 32 foram a óbito. A idade média foi de $56 \pm 16$ anos (mediana $=$ 58 , intervalo $=17-87$ ). Quanto aos hábitos de vida, foi reportado uso de álcool em 28 , de drogas em 3 e de fumo em 30 pacientes. O período médio de internação foi de $34 \pm 29$ dias (mediana $=27, \mathrm{IQ}=24$, intervalo $=2-165$ ). A média de albumina humana usada por paciente foi de 15 \pm 24 unidades (mediana $=6, \mathrm{IQ}=11$, intervalo $=$ 1-160) para tratamento médio de $5 \pm 7$ dias (mediana $=2, I Q=4$, intervalo $=1-37$, que corresponde a uma dose diária média em gramas por paciente de $30 \pm 18 \mathrm{~g}$ (mediana $=28$, IQ $=10$, intervalo $=10-120)$

As clínicas de onde se originaram mais prescrições de albumina humana foram a cirurgia geral, a clínica médica, a cardiologia e a cirurgia vascular. Houve discrepância entre o consumo registrado nas clínicas e aquele do Serviço de Farmácia, o último tendo dispensado 1.540 unidades e sendo a utilização efetiva registrada nos prontuários de 1.475 unidades. Somente a cirurgia geral foi responsável pela diferença negativa de $50(76,9 \%)$ das 65 unidades cujo consumo não foi localizado. Observa-se que diversas clínicas encaminharam ao Serviço de Farmácia menos prescrições do que as que constam nos prontuários. Por exemplo, a cardiologia solicitou albumina humana para 106 pacientes mas, segundo os prontuários, utilizou o produto em 118 pacientes (Tabela 3).

Os 99 pacientes, cujos dados foram coletados em prontuário, receberam albumina humana para 139 indicações terapêuticas. O nú- 
Tabela 1

Síntese dos protocolos de uso de albumina humana 7,8,20,23.

Usos apropriados (evidências suficientes de efetividade)

Cirurgia cardiopulmonar com circulação extracorpórea

Paracentese/ascite/cirrose

Plasmaferese

Grandes queimaduras

Ressecção hepática/transplante hepático

Síndrome nefrótica/nefrose aguda
- "prime" da bomba de circulação extracorporal

- contra-indicação de colóide não protéico

- remoção > 4 litros de líquido ascítico

- ascites diurético-refratárias

- paracenteses evacuadoras de ascites neoplásicas

- trocas plasmáticas $>20 \mathrm{ml} / \mathrm{kg} / 1$ sessão ou $>20 \mathrm{ml} / \mathrm{kg} / \mathrm{semana}$ em sessões repetidas

- 24 a 48 horas após grandes queimaduras com perda protéica importante, depleção severa de albumina

- terapia com cristalóide não efetiva

- pressão de dióxido de carbono $<20$; albumina $<2,5 \mathrm{~g} / \mathrm{dl}$

- contra-indicação de colóide não protéico

- pré e intra-operatório em pressão coloidosmótica $<12 \mathrm{mmHg}$,

pós-operatório imediato

- albuminemia $<2,5 \mathrm{~g} / \mathrm{dl}$

- hematócrito > 30\%

- falência hepática

- com diurético de alça

- albuminemia $<2,0 \mathrm{~g} / \mathrm{dl}$

- edema diurético resistente, falência renal

- edema pulmonar agudo, derrame pleural, pericárdico ou ascite

Usos controvertidos ou que necessitam de mais estudos

(evidências insuficientes de efetividade)

Cirurgia abdominal radical

Desintoxicação/hiperbilirrubinemia

Pancreatite severa necrosante

Síndrome de angústia respiratória do adulto

Síndrome de hiperestimulação ovariana
Exceções apropriadas:

- intra-operatório com albuminemia $<3,0 \mathrm{~g} / \mathrm{dl}$

- pós-operatório com hipoalbuminemia severa não corrigida por nutrição parenteral

- antes ou após ex-sangüíneo em neonatos

- não há evidências conclusivas em transporte de proteínas em adultos

- efetivo na prática - não há evidências conclusivas de efetividade

Exceções apropriadas:

- contra-indicação de colóide não protéico

- quantidade máxima de colóide não protéico excedida

- como prevenção durante ou no dia da coleta do óvulo

- efetivo na prática - não há evidências conclusivas de efetividade

\section{Usos inapropriados (não há evidências de efetividade)}

Choque hipovolêmico (hemorrágico, não hemorrágico, séptico)

Isquemia cerebral

\section{Pancreatite}

Reposição nutricional/hipoalbuminemia

Transplante (exceto hepático)
Exceções apropriadas:

- necessidade de restrição de sódio

- contra indicação de CNP, dose de CNP excedida

- grávidas

- pressão sistólica $<80 \mathrm{mmHg}$, pressão venosa central $<6 \mathrm{~cm} \mathrm{H2O}$, pressão capilar pulmonar $<10 \mathrm{mmHg}$ e/ou pressão coloidosmótica $<15 \mathrm{mmHg}$

- não recomendado para hemodiluição

- não há evidências de efetividade

- não indicado como repositor de albumina ou fonte protéica

Exceções apropriadas:

- diarréia (> 2l/dia), hipoalbuminemia $(<2,0 \mathrm{~g} / \mathrm{dl})$

- intolerância de nutrição enteral

- intra e pós-operatório de transplante renal - não há evidência de efetividade Exceção apropriada:

- pâncreas - doador e receptor 
Perdas de prontuários selecionados e excluídos da análise - março a agosto de 2001 .

\begin{tabular}{lrr}
\hline Motivos de perda & Pacientes & Unidades \\
\hline Prescrição atendida pela farmácia e suspensa (não usou albumina humana) & 5 & 13 \\
Prontuário não localizado pelo Serviço de Documentação Científica & 6 & 56 \\
Prontuário não devolvido ao Serviço de Documentação Científica & 2 & 44 \\
Internação no período não arquivada no prontuário & 16 & 155 \\
Total & 16 \\
\hline
\end{tabular}

mero não corresponde ao de pacientes porque alguns receberam albumina humana para mais de uma indicação (Tabela 4). Não foi possível determinar, pelos registros dos prontuários, o motivo da indicação para dois pacientes.

As 29 (20,9\%) indicações classificadas como outras correspondem àquelas que não estão referidas nos protocolos utilizados, como por exemplo, cirurgia cardíaca sem circulação extracorpórea $(n=4)$ e septicemia $(n=3)$. Entretanto, em 16 pacientes não era a única indicação, o que resulta numa freqüência de 13 (9,3\%) indicações únicas classificadas como outras.

Em 23 (16,5\%) indicações de albumina humana para correção de hipoalbuminemia/reposição nutricional, apenas $10(43,5 \%)$ foram prescritas para pacientes com nível crítico de albuminemia $(<2,0 \mathrm{~g} / \mathrm{l})$.

Foram encontrados dois relatos de suspeitas de reações adversas. Um paciente apresentou defeito de coagulação (petéquias) e hipotensão. O outro apresentou hipersensibilidade (febre $39,5^{\circ} \mathrm{C}$, tremores, calafrios, elevação da pressão arterial), sugerindo reação pirogênica.

Após a análise da adequação, 165 prescrições $(33,1 \%)$ foram consideradas apropriadas, $308(61,8 \%)$ inapropriadas e $23(4,6 \%)$ controversas, restando $2(0,4 \%)$ indeterminadas por carência de dados no prontuário. A proporção de inadequações corresponde a 800 unidades de $50 \mathrm{ml}$ a $20 \%$, equivalentes a $8.000 \mathrm{~g}(61,8 \%)$ de albumina humana (Tabela 5).

A cirurgia geral foi o setor responsável pela maior proporção de inadequações, correspondentes a 189 (38,0\%) prescrições e 499 unidades $(33,8 \%)$. A clínica médica apresentou a menor proporção de inadequação, com 15,5\% (n = 16) das prescrições dessa clínica $(n=103)$ classificadas como inapropriadas. Foi encontrada associação significativa $(\mathrm{p}<0,01)$ entre o tipo de clínica (cirúrgicas e não cirúrgicas) e a freqüência de prescrições inapropriadas.

\section{Discussão}

O presente estudo analisou criticamente as características de utilização da albumina humana em um hospital da rede pública no Rio de Janeiro, entre março e agosto de 2001. Os 99 pacientes incluídos no estudo têm uma distribuição equilibrada quanto ao sexo e idade média de 56 anos. As clínicas que mais prescreveram e consumiram albumina humana foram a cirurgia geral, a cardiologia e a clínica médica.

Cerca de $62,0 \%$ das prescrições foram consideradas inadequadas. As maiores proporções de inadequação de prescrição provêm da cirurgia geral, da cardiologia e da cirurgia vascular, em termos absolutos e relativos. Embora todos os protocolos considerados não recomendem a albumina humana para reposição protéica, o estudo apontou que esta corresponde a $17,0 \%$ das indicações, das quais $56,0 \%$ não apresentavam hipoalbuminemia severa $(<2,0 \mathrm{~g} / \mathrm{dl})$.

A associação encontrada entre as clínicas cirúrgicas (cardiologia, cirurgia geral, cirurgia vascular e ortopedia) e a inadequação de uso de albumina humana confirma os achados de outros estudos 16,19 e indica a necessidade de concentrar as intervenções educativas nesses setores.

Os resultados mostram que as $8.000 \mathrm{~g}$ de albumina humana usadas inadequadamente totalizam gastos da ordem de US\$ 16 mil a US\$ 20 mil. Esse montante poderia ser reduzido, tanto nos casos em que não haveria necessidade de qualquer expansor plasmático, como naqueles em que poderia ter sido empregado um substituto terapêutico de custo mais baixo.

O presente estudo encontrou resultados compatíveis com os estudos de outros países. Entretanto, os dados obtidos estão possivelmente subestimados uma vez que não foram incluídos os pacientes do CTI e da emergência, que consumiram, juntos, $44,0 \%$ do total de unidades do produto dispensadas pelo Serviço de Farmácia no período analisado. Optamos por 


\section{Tabela 3}

Uso de albumina humana segundo fonte de informação, por clínica março a agosto de 2001.

\begin{tabular}{|c|c|c|c|}
\hline Clínica & Prontuários (n) & Farmácia (n) & $\begin{array}{c}\text { Diferença entre } \\
\text { prontuários e } \\
\text { farmácia (\%) }\end{array}$ \\
\hline \multicolumn{4}{|l|}{ Cirurgia geral } \\
\hline Unidades & 606 & 656 & $-7,6$ \\
\hline Prescrições & 229 & 235 & $-2,6$ \\
\hline \multicolumn{4}{|l|}{ Clínica médica } \\
\hline Unidades & 448 & 457 & $-2,0$ \\
\hline Prescrições & 103 & 94 & $+9,6$ \\
\hline \multicolumn{4}{|l|}{ Cardiologia } \\
\hline Unidades & 314 & 316 & $-0,6$ \\
\hline Prescrições & 118 & 106 & $+11,3$ \\
\hline \multicolumn{4}{|c|}{ Cirurgia vascular } \\
\hline Unidades & 56 & 54 & $+3,7$ \\
\hline Prescrições & 22 & 18 & $+22,2$ \\
\hline \multicolumn{4}{|l|}{ Ginecologia } \\
\hline Unidades & 25 & 26 & $-3,8$ \\
\hline Prescrições & 15 & 15 & 0,0 \\
\hline \multicolumn{4}{|l|}{ Nefrologia } \\
\hline Unidades & 15 & 15 & 0,0 \\
\hline Prescrições & 5 & 5 & 0,0 \\
\hline \multicolumn{4}{|l|}{ Obstetrícia } \\
\hline Unidades & 7 & 6 & $+16,7$ \\
\hline Prescrições & 3 & 2 & $+50,0$ \\
\hline \multicolumn{4}{|c|}{ Diálise Peritonial } \\
\hline Unidades & 2 & 4 & $-50,0$ \\
\hline Prescrições & 1 & 2 & $-50,0$ \\
\hline \multicolumn{4}{|l|}{ Hemodiálise } \\
\hline Unidades & 1 & 4 & $-75,0$ \\
\hline Prescrições & 1 & 2 & $-50,0$ \\
\hline \multicolumn{4}{|l|}{ Ortopedia } \\
\hline Unidades & 1 & 2 & $-50,0$ \\
\hline Prescrições & 1 & 1 & 0,0 \\
\hline \multicolumn{4}{|l|}{ Total } \\
\hline Unidades & 1.475 & 1.540 & $-4,2$ \\
\hline Prescrições & 498 & 480 & $+3,8$ \\
\hline
\end{tabular}

excluir o CTI porque, para a análise do uso de albumina humana em pacientes críticos, polimedicados e com quadros clínicos complexos, seria necessária a participação de painel de especialistas. A emergência foi excluída devido à inexistência de prontuário da maioria dos pacientes e a possibilidade de perdas diferenciadas, que poderia enviesar o estudo.

Em 1993, o Clinical Practice Advencement Center, ligado ao University Hospital Consortium (UHC), dos Estados Unidos, desenvolveu um protocolo para o uso apropriado de soluções de albumina humana, de colóides não protéicos e de cristalóides. Isso porque no ano anterior os gastos com albumina humana variaram entre US $\$ 60$ mil e US $\$ 800$ mil, por hospital membro do UHC, e havia a necessidade de incorporar os avanços de conhecimento pós 1975, ano da elaboração do protocolo NIH 7. Cerca de quatro meses após a distribuição do protocolo, sem que se fizessem esforços educativos para o seu emprego, foi realizado um estudo observacional prospectivo em 15 hospitais do UHC. Os resultados mostraram que no total de 982 prescrições, $24,0 \%$ eram apropriadas e $76,0 \%$ inapropriadas 19 .

Em 2000, o UHC desenvolveu um novo protocolo de albumina humana e substitutos, incorporando as evidências científicas surgidas desde 1993. Alguns meses após foi realizado um estudo de utilização de medicamento, com o intuito de avaliar o padrão de uso de albumina humana em 53 hospitais do UHC e da Voluntary Hospital Association. A média de inadequação entre clínicas foi de 57,8\% em adultos e de $52,2 \%$ em crianças 16 .

Um estudo observacional prospectivo, realizado em dois hospitais universitários na Espanha, avaliou a adequação da prescrição e a repercussão econômica do uso inapropriado. O protocolo de referência foi o publicado em 1995 por Vermeulen et al. 7. Foram analisadas as prescrições de albumina de 197 pacientes, que receberam um total de 3.208 frascos de $50 \mathrm{ml}$ de albumina humana $20 \%$. Apenas $8,1 \%$ das prescrições foram consideradas apropriadas. Mais de 90,0\% dos frascos foram consumidos em prescrições inapropriadas, o que acarretou um impacto de custos importante 13.

O Grupo Andaluz para Estúdios de Utilización de Medicamentos desenvolveu, em 1997, um protocolo de consenso, avaliado posteriormente em 22 hospitais públicos da Espanha. Em 242 prescrições foram consumidas $62.282 \mathrm{~g}$ do produto. Somente $23,0 \%$ foram consideradas apropriadas. O custo do uso inapropriado foi de US $\$ 140.891,00(77,0 \%)$. Os resultados sugerem melhoria nos padrões de prescrição, 
quando comparados com os obtidos no estudo de Vargas et al. 13, desenvolvido antes da disseminação do protocolo, embora tenham sido realizados com instituições e profissionais diferentes 8 .

Um estudo de 1996 num hospital de 850 leitos, na França, comparou os níveis de consumo e os padrões de prescrição, antes e após a difusão de protocolo de albumina humana. Inicialmente, houve $25,5 \%$ de adequação e $38,5 \%$ de inadequação; após a difusão do protocolo, a proporção de adequação elevou-se para 47,5\% e a de inadequação caiu para $14,5 \%$. O processo de avaliação foi mantido nos meses subseqüentes e a proporção de adequação atingiu $59,0 \%$, sendo o restante, prescrições controversas ou com poucas evidências e não houve mais prescrições inapropriadas. A redução de consumo durante o período do estudo foi da ordem de $60,0 \%$, indicando a importância do
Indicações de uso de albumina humana - março a agosto de 2001.

\begin{tabular}{lrr}
\hline Indicações & $\mathbf{n}$ & $\%$ \\
\hline Reposição nutricional/hipoalbuminemia & 23 & 16,5 \\
Paracentese & 21 & 15,1 \\
Cirurgia cardiopulmonar com circulação extracorpórea & 18 & 12,9 \\
Cirurgia abdominal radical & 15 & 10,8 \\
Cirrose crônica & 12 & 8,6 \\
Nefrose aguda & 7 & 5,0 \\
Nefrose crônica & 6 & 4,3 \\
Choque hemorrágico & 3 & 2,2 \\
Choque não hemorrágico & 2 & 1,4 \\
Isquemia cerebral & 2 & 0,7 \\
Pancreatite & 2 & 1,4 \\
Outras & 29 & 20,9 \\
Total & 139 & 100,0
\end{tabular}

Adequação de uso de albumina humana por clínicas - março a agosto de 2001.

\begin{tabular}{|c|c|c|c|c|c|c|c|c|c|c|}
\hline \multirow[t]{3}{*}{ Clínica } & \multicolumn{10}{|c|}{ Uso } \\
\hline & \multicolumn{2}{|c|}{ Apropriado } & \multicolumn{2}{|c|}{ Inapropriado } & \multicolumn{2}{|c|}{ Controverso } & \multicolumn{2}{|c|}{ Indeterminado } & \multicolumn{2}{|c|}{ Total } \\
\hline & $n$ & $\%$ & $n$ & $\%$ & $n$ & $\%$ & $\mathrm{n}$ & $\%$ & $\mathrm{n}$ & $\%$ \\
\hline \multicolumn{11}{|l|}{ Cirurgia geral } \\
\hline Unidades & 53 & 3,6 & 499 & 33,8 & 51 & 3,5 & 3 & 0,2 & 606 & 41,1 \\
\hline Prescrições & 19 & 3,8 & 189 & 38,0 & 20 & 4,0 & 1 & 0,2 & 229 & 46,0 \\
\hline \multicolumn{11}{|l|}{ Clínica médica } \\
\hline Unidades & 397 & 26,9 & 49 & 3,3 & 0 & 0,0 & 2 & 0,1 & 448 & 30,4 \\
\hline Prescrições & 86 & 17,3 & 16 & 3,2 & 0 & 0,0 & 1 & 0,2 & 103 & 20,7 \\
\hline \multicolumn{11}{|l|}{ Cardiologia } \\
\hline Unidades & 146 & 9,9 & 168 & 11,4 & 0 & 0,0 & 0 & 0,0 & 314 & 21,3 \\
\hline Prescrições & 54 & 10,8 & 64 & 12,9 & 0 & 0,0 & 0 & 0,0 & 118 & 23,7 \\
\hline \multicolumn{11}{|c|}{ Cirurgia vascular } \\
\hline Unidades & 0 & 0,0 & 56 & 3,8 & 0 & 0,0 & 0 & 0,0 & 56 & 3,8 \\
\hline Prescrições & 0 & 0,0 & 22 & 4,4 & 0 & 0,0 & 0 & 0,0 & 22 & 4,4 \\
\hline \multicolumn{11}{|l|}{ Outras } \\
\hline Unidades & 16 & 1,1 & 28 & 1,9 & 7 & 0,5 & 0 & 0,0 & 51 & 3,5 \\
\hline Prescrições & 6 & 1,2 & 17 & 3,4 & 3 & 0,6 & 0 & 0,0 & 26 & 5,2 \\
\hline \multicolumn{11}{|l|}{ Total } \\
\hline Unidades & 612 & 41,5 & 800 & 54,2 & 58 & 4,0 & 5 & 0,3 & 1.475 & 100,0 \\
\hline Prescrições & 165 & 33,1 & 308 & 61,8 & 23 & 4,6 & 2 & 0,4 & 498 & 100,0 \\
\hline
\end{tabular}


envolvimento dos profissionais de saúde, especialmente do corpo clínico, na elaboração e implantação do protocolo, o que aumenta a aceitabilidade e os impactos positivos 20.

A albumina humana é um hemoderivado considerado mal utilizado na prática clínica, em desacordo com as diretrizes dos protocolos internacionalmente aceitos. As revisões Cochrane indicaram a necessidade de estimular a realização de estudos que produzam evidências consistentes para o uso racional da albumina humana e dos substitutos terapêuticos 24 .

Entretanto, a simples divulgação de um protocolo tem pouco impacto e, para que o protocolo divulgado pela ANVISA 23 possa se converter em um instrumento efetivo de racionalização terapêutica, sua implantação deve vir acompanhada por estratégias ativas e específicas de disseminação, e por estudos de avaliação de efetividade e eficiência 25 .

Encontramos dois relatos de suspeitas de reações adversas. O monitoramento dos efeitos adversos atribuídos ao uso de hemoderivados integra o campo da farmacovigilância, porque são medicamentos 5 . As reações adversas à administração intravenosa de albumina humana são pouco freqüentes, de diversas naturezas, algumas graves 25 . Os relatos encontrados de suspeitas de reações adversas não foram avaliados quanto à causalidade, mas descrevem sintomas compatíveis com os apontados pela literatura. Se confirmadas, indicariam que $2,0 \%$ dos pacientes apresentaram reação adversa (reações freqüentes: $\geq 1,0 \% \mathrm{a}<10,0 \% 26$ ). $\mathrm{O}$ achado aponta para a importância do monitoramento intensivo dos eventos adversos durante o uso de albumina humana.

Consideramos os resultados obtidos confiáveis e válidos. A avaliação da concordância quanto à indicação terapêutica numa subamostra revelou uma boa confiabilidade.

Houve discordância entre os registros da farmácia e os dos prontuários quanto às unidades do produto consumidas. Esse fato não interferiu nos resultados do estudo, uma vez que as informações basearam-se nos prontuários. Entretanto, sugere possíveis falhas no controle interno do hospital.

Outro problema foi a inexistência do número de matrícula dos pacientes nas prescrições encaminhadas à farmácia, o que dificultou a localização dos prontuários. As perdas do estudo não parecem introduzir vieses por não serem diferenciadas quanto às características dos pacientes, das clínicas e da terapêutica, em relação aos casos incluídos.

Os procedimentos adotados para a calibração do instrumento de medida (Formulário de Coleta de Dados de Prontuário), durante o préteste e o estudo piloto, levaram a melhorias no manual de instruções e no treinamento da equipe. A elaboração do instrumento, em particular nas indicações terapêuticas e sinais complementares, contou com o suporte de especialista clínico de hospital de referência, externo ao hospital selecionado.

Sugerimos a repetição do estudo em outros hospitais para estabelecer comparações e para definir melhor os padrões de utilização da albumina humana e seus determinantes. Da mesma forma, estudos prospectivos sobre a prescrição de albumina humana e expansores sintéticos, com a participação da Comissão de Farmácia e Terapêutica e de painel de especialistas, podem trazer importantes contribuições para a racionalização do uso do produto, inclusive incorporando o componente custo na avaliação.

\section{Resumo}

A albumina humana é freqüentemente usada sem que haja correspondência entre suas propriedades farmacológicas e as doenças. Este é um estudo das prescrições de albumina humana em um hospital da rede pública no Rio de Janeiro, Brasil, com ênfase no grau de adesão das mesmas a protocolos de tratamento. As informações foram extraídas dos registros do Serviço de Farmácia e dos prontuários médicos de 99 pacientes adultos, que receberam, entre março e agosto de 2001, 1.475 unidades de albumina humana, solicitadas em 498 prescrições. A análise de confiabilidade das informações sobre indicação terapêutica resultou num kappa de 0,79 (IC95\%: 0,72-0,85). As indicações terapêuticas foram avaliadas como apropriadas, inapro- priadas ou controversas, com base em quatro protocolos de países diferentes. Do total de prescrições, 33,1\% foram consideradas apropriadas, 61,8\% inapropriadas, 4,6\% controversas e $0,4 \%$ indeteminadas. Identificaram-se dois casos suspeitos de reações adversas. A proporção de prescrições inapropriadas é preocupante, dado o elevado custo do produto e o seu potencial para causar reações adversas. A implantação das diretrizes nacionais expressas no protocolo da ANVISA é estratégica para a promoção do uso racional da albumina humana em hospitais.

Albumina Sérica; Uso de Medicamentos; Avaliação dos Serviços 


\section{Colaboradores}

G. C. Matos fez a revisão da literatura, executou o trabalho de campo, realizou a análise de dados e a redação do trabalho. S. Rozenfeld concebeu o estudo e o desenho, orientou a coleta e a análise de dados e participou da redação do texto.

\section{Referências}

1. Sena JL. Cuidados intensivos 600.000 litros en exceso. Se demuestra que el tratamiento con albúmina causa más problemas que beneficios. http: //www.el-mundo.es/salud/1998/306/02082.html (acessado em 13/Mai/2001).

2. Agência Nacional de Vigilância Sanitária. Registro de produtos. http://www.anvisa.gov.br/medicamentos/registro (acessado em 01/Fev/2003).

3. Ministério da Saúde. Sistema de Informações Hospitalares. Procedimentos especiais. http:// dtr2001.saude.gov.br/sas/decas/pesqdescresp.asp (acessado em 10/Jul/2004).

4. Agência Nacional de Vigilância Sanitária. Programa Nacional de Hemoderivados. http://www.anvisa.gov.br/sangue (acessado em 30/Jul/2003).

5. Lesne-Hulin A, Bourget $\mathrm{Ph}$, Silvie M, Barath V, Singlas E. Traçabilité des médicaments dérivés du sang: réglementation et implications pharmacoéconomiques après 24 mois d'exercice dans un CHU parisien. Path Biol (Paris) 1997; 45:741-50.

6. Sweetman SC. Martindale: the complete drug reference [CD-ROM]. London: Pharmaceutical Press/ Micromedex Inc.; 2001.

7. Vermeulen LC, Ratko TA, Erstad BL, Brecher ME, Matuszewski KA. A paradigm for consensus: the University Hospital Consortium Guidelines for the use of albumin, nonprotein colloid, and crystalloid solutions. Arch Intern Med 1995; 155:373-9.

8. Remohi MJT, Arcos AS, Ramos BS, Paloma JB, Aznar MDG. Costs related to inappropriate use of albumin in Spain. Ann Pharmacother 2000; 34: 1198-205.

9. Bunn F, Alderson P, Hawkins V. Colloid solution for fluid resuscitation. (Cochrane Review). In: The Cochrane Library, Issue 4, 2004. Oxford: Update Software.

10. Roberts I, Alderson P, Bunn F, Chinnock P, Ker K, Schierhout G. Colloids versus crystalloids for fluid resuscitation in critically ill patients. (Cochrane Review). In: The Cochrane Library, Issue 4, 2004. Oxford: Update Software.

11. Alderson P, Bunn F, Lefebvre C, Li Wan Po A, Li L, Roberts I, et al. The albumin revierwers. Human albumin solution for resuscitation and volume expansion in critically ill patients. (Cochrane Review). In: The Cochrane Library, Issue 4, 2004. Oxford: Update Software.

12. Finfer S, Bellomo R, Boyce N, French J, Myburgh J, Norton R, et al. A comparison of albumin and saline for fluid resuscitation in the intensive care unit. N Eng J Med 2004; 350:2247-56.

13. Vargas E, de Miguel V, Portoles A, Avendaño C,

\section{Agradecimentos}

Agradecemos ao Dr. Luiz Amorim (HEMORIO) pelas críticas e sugestões; a Isabel Cristina Martins Emmerick e Durval Martins Pontes Jr., pela coleta dos dados; a Haroldo Leal Cazes, pela criação e arte do formulário. À Coordenação de Pós-graduação (Escola Nacional de Saúde Pública) pela contratação dos coletadores e à Secretaria de Estado de Saúde do Rio de Janeiro pela bolsa de auxílio à pesquisa concedida à autora principal.

Ambit MI, Torralba A, et al. Use of albumin in two Spanish university hospitals. Eur J Clin Pharmacol 1997; 52:465-70.

14. Tullis JL. Albumin. 1. Background and use. JAMA 1977; 237:355-9.

15. Tullis JL. Albumin. 2. Guidelines for clinical use. JAMA $1977 ; 237: 460-3$.

16. Tanzi M, Gardner M, Megellas M, Lucio S, Restino M. Evaluation of the appropriate use of albumin in adult and pediatric patients. Am J Health Syst Pharm 2003; 60:1330-5

17. Woolf SH. Evidence-based medicine and practice guidelines - an overview. Cancer Control 2000; 7:362-7.

18. Scott IA, Buckmaster ND, Harvey KH. Clinical practice guidelines: perspectives of clinicians in Queensland public hospitals. Intern Med J 2003; 33:273-9.

19. Yim JM, Vermeulen LC, Erstad BL, Matuszewski KA, Burnett DA, Vlasses PH. Albumin and nonprotein colloid solution use in US Academic Health Centers. Arch Intern Med 1995; 155:2450-5.

20. Debrix I, Combeau D, Stephan F, Benomar A, Becker A. Clinical practice guidelines for the use of albumin: results of a drug use evaluation in a Paris hospital. Pharm World Sci 1999; 21:11-6.

21. Hospital Israelita Albert Einstein. Manual de administração de sangue, componentes, derivados, fatores de crescimento de medula óssea e de aféreses terapêuticas. São Paulo: Hospital Israelita Albert Einstein; 1997.

22. Ribeiro E. Avaliação do uso de um instrumento educativo para solicitação de albumina humana em um hospital universitário de São Paulo [Tese de Doutorado]. São Paulo: Faculdade de Ciências Farmacêuticas, Universidade de São Paulo; 2001.

23. Agência Nacional de Vigilância Sanitária, Ministério da Saúde. Resolução de Diretoria Colegiada RDC n. 115. Aprova as diretrizes para o uso de albumina. Diário Oficial da União 2004; 11 mai.

24. Erstad BL. Concerns with defining appropriate uses of albumin by meta-analisys. Am J Health Syst Pharm 1999; 56:1451-4.

25. Gonzalez ER, Kannwurf B. The clinical use of albumin. US Pharm 1998; 23:HS15-HS26.

26. Uppsala Monitoring Centre, World Health Organization. Definitions. http://www.who-umc.org/ index2.html (acessado em 04/Jul/2004).

Recebido em 02/Ago/2004

Versão final reapresentada em 27/Jan/2005

Aprovado em 14/Mar/2005 